Rev. Adm. Saúde (On-line), São Paulo, v. 19, n. 74, jan. - mar. 2019, Epub 18 mar. 2019 http://dx.doi.org/10.23973/ras.74.158

RELATO DE CASO

\title{
Atendimento ambulatorial dos pacientes em terapia de anticoagulação de um hospital cardiológico de alta complexidade do município de São Paulo
}

Ambulatory care of patients in anticoagulation therapy in a high complexity cardiology hospital in São Paulo city

Ticiane Nishimoto', Eric Magalhães de Moraes², Eduardo Ryoiti Tatebe ${ }^{3}$, Guilherme do Espírito Santo Silva ${ }^{4}$, Paula Cristina Souto de Camargo ${ }^{5}$

1. Gerontóloga, especialista em administração hospitalar e de sistemas de saúde.

Colaboradora do Programa de Estudos Avançados em Administração Hospitalar e Sistemas de Saúde (PROAHSA) do Hospital das Clínicas da Faculdade de Medicina da USP (HCFMUSP).

2. Médico. Pós-graduando em administração hospitalar e sistemas de saúde pela Escola de Administração de Empresas de São Paulo (EAESP-FGV). São Paulo SP.

3. Médico. Residente do PROAHSA. São Paulo SP.

4. Médico, mestre em gestão. Superintendente assistencial da Rede Primavera Saúde. São Paulo SP.

5. Especialista em administração hospitalar e de sistemas de saúde. Coordenadora de Ensino do PROAHSA do HCFMUSP. São Paulo SP.

\section{RESUMO}

No contexto da cardiologia, os pacientes portadores de cardiopatias em uso de terapia de anticoagulação frequentemente realizam o exame de tempo de protrombina (TP) para monitorar o grau de anticoagulação do sangue e manutenção do TP para acompanhamento da medicação anticoagulante. $O$ objetivo do projeto foi analisar e propor intervenções de melhoria no processo deste atendimento ambulatorial para a realização do exame de TP dos 
pacientes em uso de terapia de anticoagulação, em um hospital público universitário de alta complexidade, especializado em cardiologia, pneumologia e cirurgias cardíaca e torácica, localizado na cidade de São Paulo. Trata-se de um estudo de caso e os dados coletados foram analisados quantitativamente em caráter exploratório, transversal e descritivo, sendo retrospectivo referente ao período de novembro de 2016 e abril de 2017. Os dados foram obtidos através de fontes internas da instituição, além de análise observacional do local e pareceres das partes envolvidas, apresentados como fase diagnóstica e proposta de intervenção. De acordo com os dados coletados, foram realizados em média 4750 exames de TP por mês, representando $25 \%$ do volume de atendimentos ambulatoriais da instituição. Aproximadamente 55\% dos pacientes estavam fora da faixa terapêutica, apresentando baixa eficácia do controle de anticoagulação. Todos os pacientes após a realização do exame de TP, passam por uma consulta breve de avaliação do médico, para analisar o resultado do exame e ajustar a medicação se necessário (ponto peculiar deste exame), apresentando um cuidado fragmentado por especialidade e centrado no atendimento médico. A partir dos dados observados, diagnóstico situacional, levantamento bibliográfico e benchmarking realizado em um hospital especializado em cardiologia de São Paulo, foi recomendada a implantação de um serviço centralizado com a criação de um "ambulatório de anticoagulação", incluindo serviços como: sistematização eletrônica específica para registro do exame de TP e conduta terapêutica; criação da tabela de anticoagulação do paciente; utilização de testes rápidos de TP do tipo; atuação da equipe multiprofissional com destaque para ações de educação em saúde e auto cuidado; serviço para dúvidas e orientações; Avaliação para introdução de novas medicações que não exijam o controle do INR.

Palavras-chave: ambulatório, cardiologia, anticoagulação, tempo de protrombina.
ABSTRACT
In the context of cardiology, patients with cardiopathies using anticoagulation therapy often perform the prothrombin time $(P T)$ test to monitor the degree of blood anticoagulation and maintenance of PT to monitor anticoagulant medication. The objective of the project was to analyze the process of this outpatient care to perform the PT examination of patients undergoing anticoagulation therapy in a high complexity public university hospital specialized in cardiology, pulmonology and cardiac and thoracic surgeries, located in the city from Sao Paulo. It is a case study and the data collected were quantitatively analyzed in an exploratory, cross-sectional and descriptive character, being retrospective for the period of November 2016 and April 2017. Data were obtained through internal sources of the institution, in addition to observational analysis of the site and opinions of the parties involved, presented as diagnostic phase and intervention proposal. According to the data collected, an average of 4750 PT examinations per month were performed, representing $25 \%$ of the institution's outpatient services. Approximately $55 \%$ of the patients were out of the therapeutic range, presenting low efficacy of anticoagulation control. All patients after the PT exam, undergo a brief evaluation of the 
physician, to analyze the result of the examination and adjust the medication if necessary (peculiar point of this examination), presenting a care fragmented by specialty and focused on the care doctor. Based on the observed data, situational diagnosis, bibliographic survey and benchmarking carried out in a specialized hospital in cardiology of São Paulo, it was recommended the implementation of a centralized service with the creation of an "anticoagulation clinic", including services such as: Specific electronic systematization for recording PT and therapeutic management; creation of the patient's anticoagulation table; use of rapid type TP tests; performance of the multiprofessional team with emphasis on health education and self-care actions; service for questions and directions; Evaluation for the introduction of new medications that do not control INR.

Keywords: ambulatory, cardiology, anticoagulation, prothrombin time.

\section{INTRODUÇÃO}

Os pacientes que fazem uso de anticoagulantes orais como a varfarina, necessitam fundamentalmente realizar o exame de tempo de protrombina (expresso pelo INR - International Normalized Ratio)', para monitorar e controlar o nível de anticoagulação do sangue. Este medicamento, em doses adequadas, é eficaz para evitar a formação de trombos no sistema circulatório (fator de risco associados as cardiomiopatias) ${ }^{2}$. Entretanto, os fármacos anticoagulantes não estão isentos de complicações, podendo levar a vários eventos de risco, como a ocorrência de hemorragia e morte, sendo classificado como um medicamento potencialmente perigoso ${ }^{1,3}$. Sem esse controle periódico, há mais riscos para o paciente de desestabilização do seu quadro.

\section{OBJETIVO}

O objetivo do projeto foi analisar e propor intervenções de melhoria no processo de atendimento aos pacientes em anticoagulação que realizam o acompanhamento do exame de tempo de protrombina (TP) no ambulatório de um hospital cardiológico de alta complexidade, especializado em cardiologia, pneumologia e cirurgias cardíaca e torácica, localizado no município de São Paulo.

\section{MÉTODO}


Trata-se de um estudo de caso, de caráter exploratório, transversal e descritivo, os dados coletados foram analisados quantitativamente no período entre novembro de 2016 e abril de 2017. Foi realizado diagnóstico situacional com análise de estrutura, processos e resultados, verificando a organização e planejamento do serviço como um todo, com o intuito de propor melhorias para a instituição e obter um melhor controle e acompanhamento do exame de anticoagulação do paciente, apresentados como fase diagnóstica e proposta de intervenção. Para embasamento das recomendações e proposta de intervenção, foi realizado um levantamento bibliográfico e benchmarking em um hospital especializado em cardiologia de São Paulo.

\section{RESULTADOS}

\section{Fase Diagnóstica}

O serviço ambulatorial destinado ao atendimento dos pacientes é composto por 40 salas de atendimento médico geral e especializado (com 17 especialidades de cardiologia e pneumologia atendendo nesse ambulatório), multiprofissional e assistência social. Recebe um fluxo médio mensal de 12 mil pacientes e considerando que os atendimentos ocorrem apenas em dias úteis, a média de atendimentos diária de 674 atendimentos registrados (fonte: relatório interno da instituição).

De acordo com o diagnóstico situacional realizado, o ambulatório recebe um grande volume de pacientes (aproximadamente 228 por dia) que vão para realização e acompanhamento do exame de tempo de protrombina (TP).

Os pacientes em uso de terapia de anticoagulação são divididos em dois horários de agendamento durante o dia (manhã e tarde), tendo pouca distribuição dos pacientes ao longo do período de funcionamento do ambulatório. Tal distribuição dos pacientes gera um impacto significativo no funcionamento do serviço ambulatorial, pois acarreta um aumento no tempo de espera em diferentes setores ambulatoriais e, consequentemente, causa uma sobrecarga de trabalho nestes períodos para os profissionais envolvidos no seu atendimento. Além disso, há um notável aumento no número de pacientes nas dependências do serviço ambulatorial, enfileiradas, causando um distúrbio no fluxo de pacientes e colaboradores. Todas essas questões tornam a experiência dos pacientes insatisfatória, o que pode ter influência na adesão do tratamento e, consequentemente, nos resultados positivos do paciente em uso de terapia de anticoagulação.

O caminho do paciente em uso de terapia anticoagulação passa pelos três setores do ambulatório. A entrada do ambulatório está localizada no Setor A (Figura 1). O paciente precisa percorrer o Setor B e, na transição com o setor $\mathrm{C}$, encontra-se o setor de coleta de exame, onde o paciente deve aguardar até ser chamado. Após coletar o exame e receber o resultado, o paciente deve se dirigir até o Setor $\mathrm{C}$, onde aguardará o atendimento médico, momento no qual o médico irá analisar o resultado do exame. Após a consulta, o paciente é encaminhado até o Setor A, para realização do agendamento do retorno 
(Figura 2). Todos os setores citados encontram-se no mesmo andar, e é possível observar o fluxo do processo na Figura 3. Como já citado anteriormente, esses pacientes compartilham a estrutura e alguns processos do ambulatório com todos os demais pacientes, o que acaba por dificultar de forma geral a experiência de todos os pacientes nesses locais.

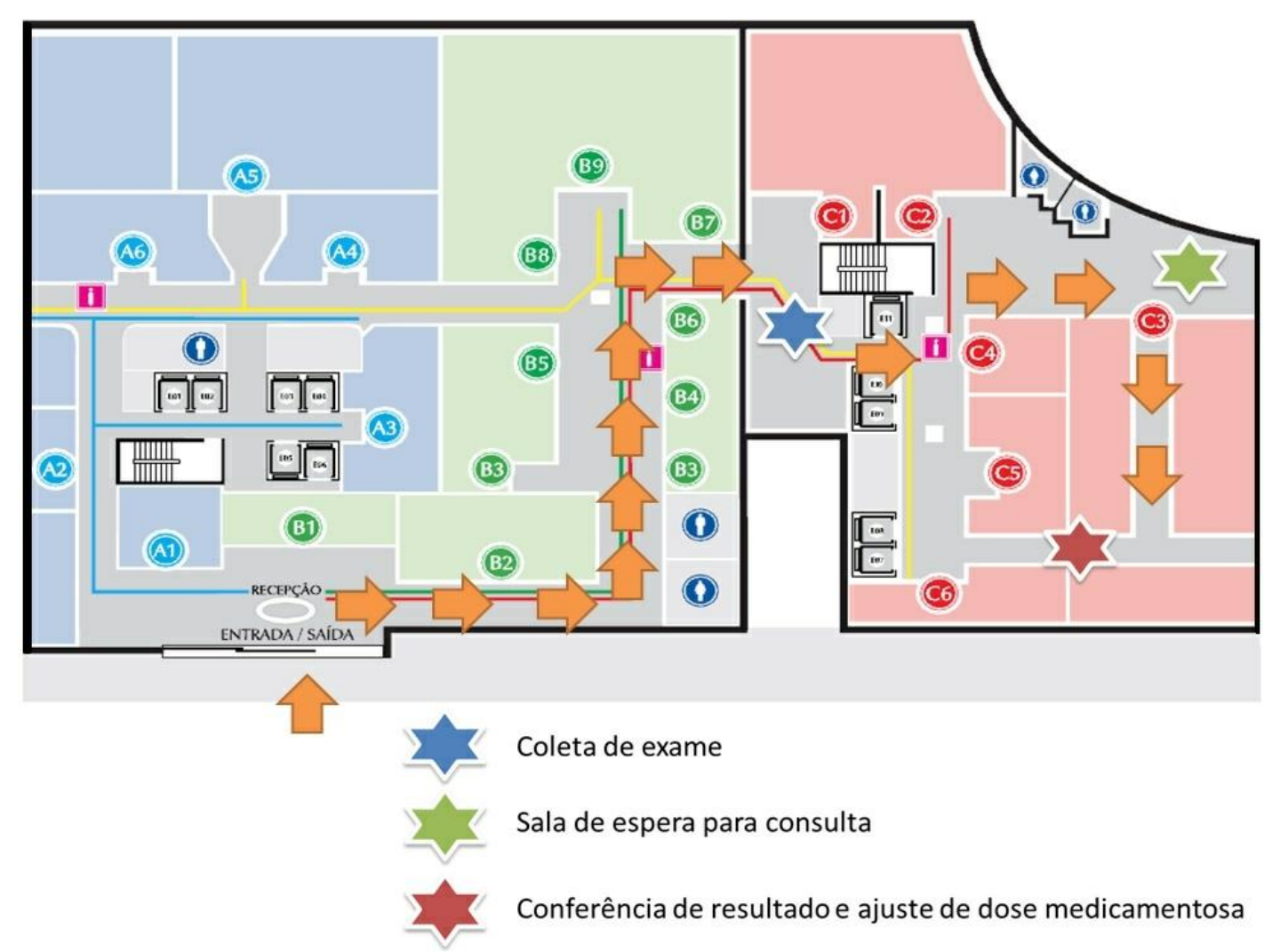

Figura 1. Mapa com o caminho de entrada do paciente. 


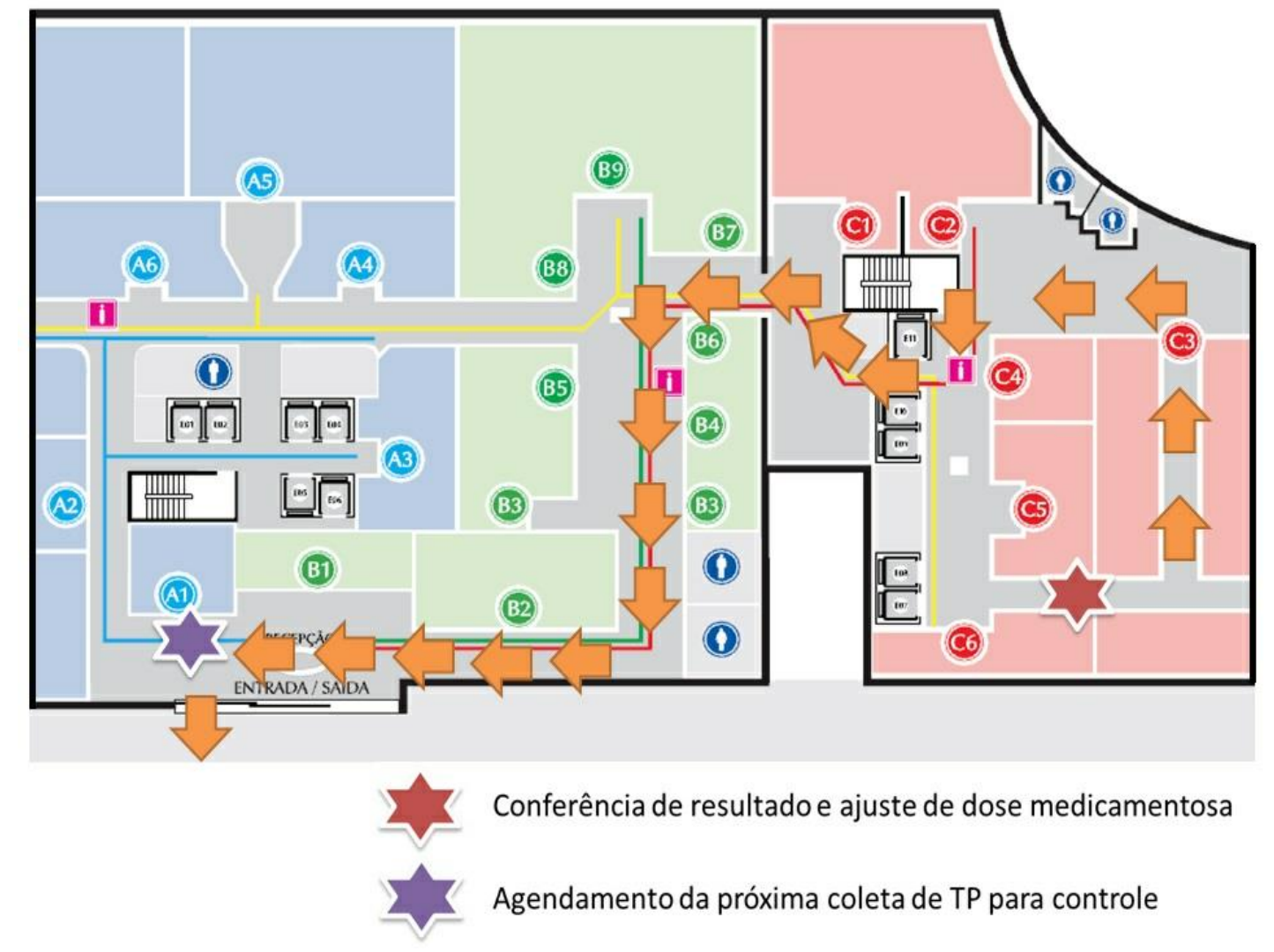

Figura 2. Mapa com o caminho de saída do paciente. 


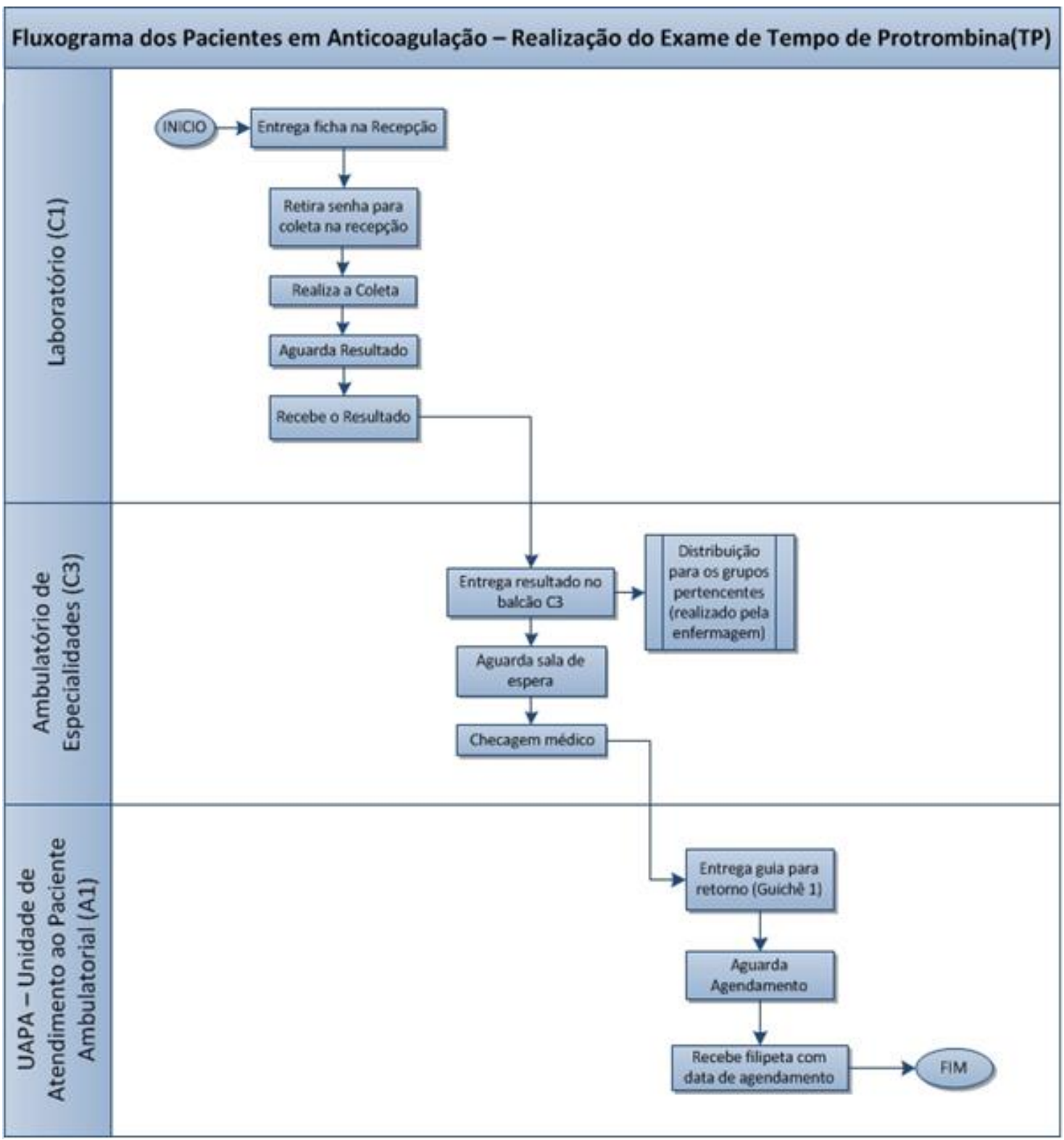

Figura 3. Fluxograma dos pacientes em anticoagulação que realizam o exame de tempo de protrombina.

Todos os pacientes em uso de terapia anticoagulação após a realização do exame do TP, passam por uma consulta breve de avaliação do médico, para analisar o resultado do exame e ajustar a medicação quando necessário, sendo este um ponto peculiar frente os demais exames realizados. Esta avaliação não é registrada dentro do prontuário eletrônico do paciente, logo não é possível ter acesso aos valores referente aos atendimentos anteriores dos pacientes. Uma vez não registrado, esse atendimento não é contabilizado oficialmente como consulta ambulatorial, e não pode ser faturado. No documento com o resultado do exame do paciente, há um campo para registrar a dosagem e posologia de anticoagulante que o paciente receberá. Além disso, as equipes médicas não dispõem de um horário pré-determinado na agenda para atendê-los, ou seja, a conferência do TP e o ajuste de medicação são 
realizados nos intervalos entre as demais consultas ambulatoriais agendadas. Dessa forma, os médicos chamam os pacientes que procuram o serviço no dia, sem ter uma previsão de quantos atendimentos de conferência de resultado de TP e ajuste de dose de medicação irão realizar no dia.

Em média, são dispensados 170.000 comprimidos de varfarina por mês pela farmácia, para os diversos institutos do hospital. Esta medicação é distribuída para as especialidades de cardiologia, reumatologia e hematologia. $O$ custo do comprimido de varfarina de $5 \mathrm{mg}$ é de aproximadamente $R \$ 0,1150$ por unidade, e em torno de $60 \%$ dos pacientes recebem a medicação em casa.

Em relação ao número total de exames realizados de TP (Gráfico 1), nos últimos seis meses (período entre novembro de 2016 e abril de 2017), foram realizados em média 4750 atendimentos por mês, incluindo os atendimentos pelo SUS - Sistema Único de Saúde, saúde suplementar e internações de pacientes não críticos, tendo uma grande parcela deste atendimento pelo SUS. A média diária é de 228 atendimentos por dia.

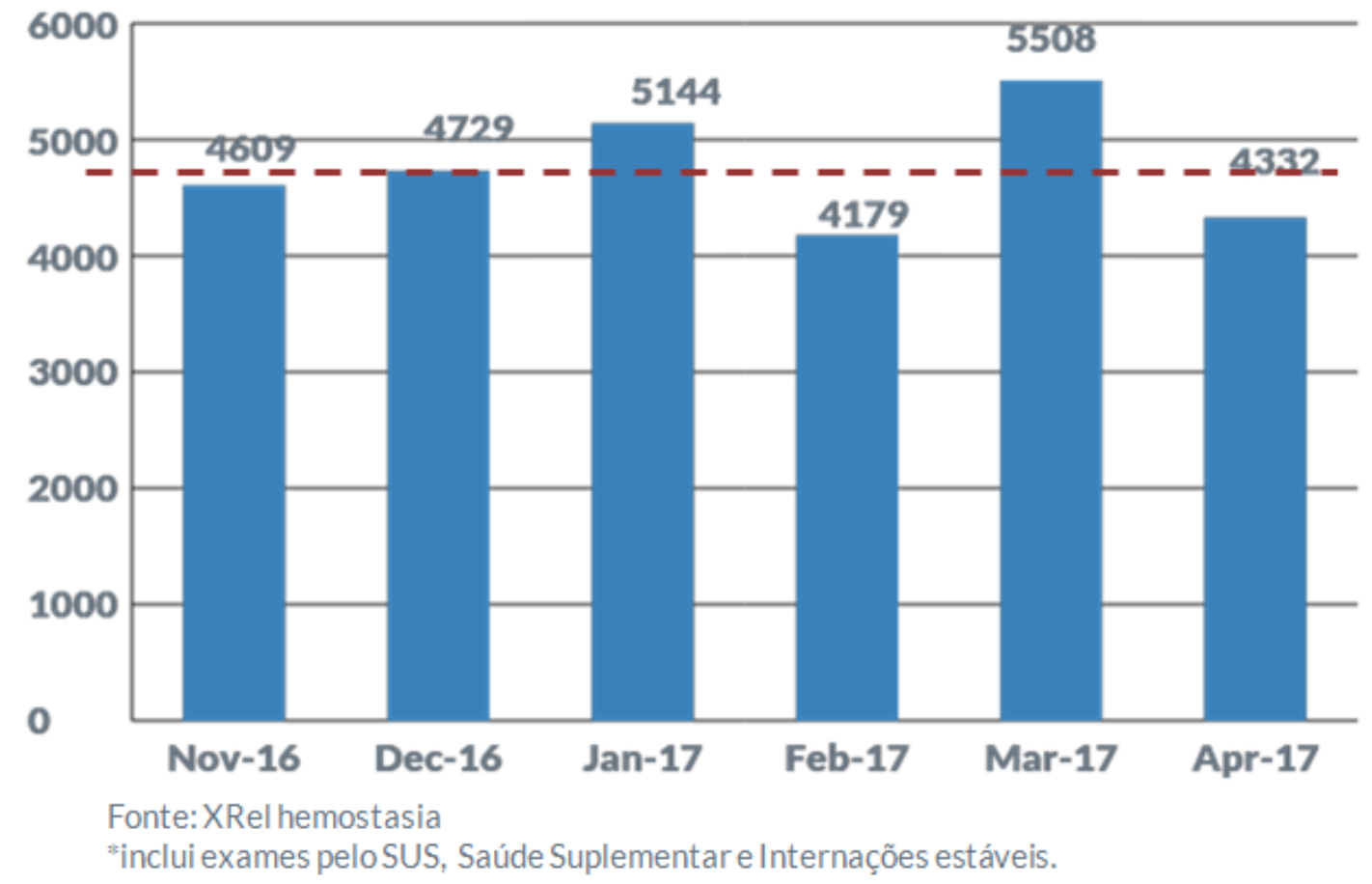

Gráfico 1. Média mensal de exames de tempo de protrombina (inclui exames realizado pelo SUS, Saúde Suplementar e Internações não críticas).

Em relação ao número total de exames de TP realizados no primeiro trimestre de 2017, distribuídos pelas especialidades (Gráfico 2), as equipes que possuem maior volume de atendimentos de TP são a arritmia (com 725 atendimentos), válvula (com 722 atendimentos) e equipe de marca-passo (com 466 atendimentos). Estes dados representam apenas os pacientes agendados, não incluso o total de pacientes por equipe especializada, pois o controle sobre 
a realização do exame não é centralizado e não são todas as equipes que o fazem.

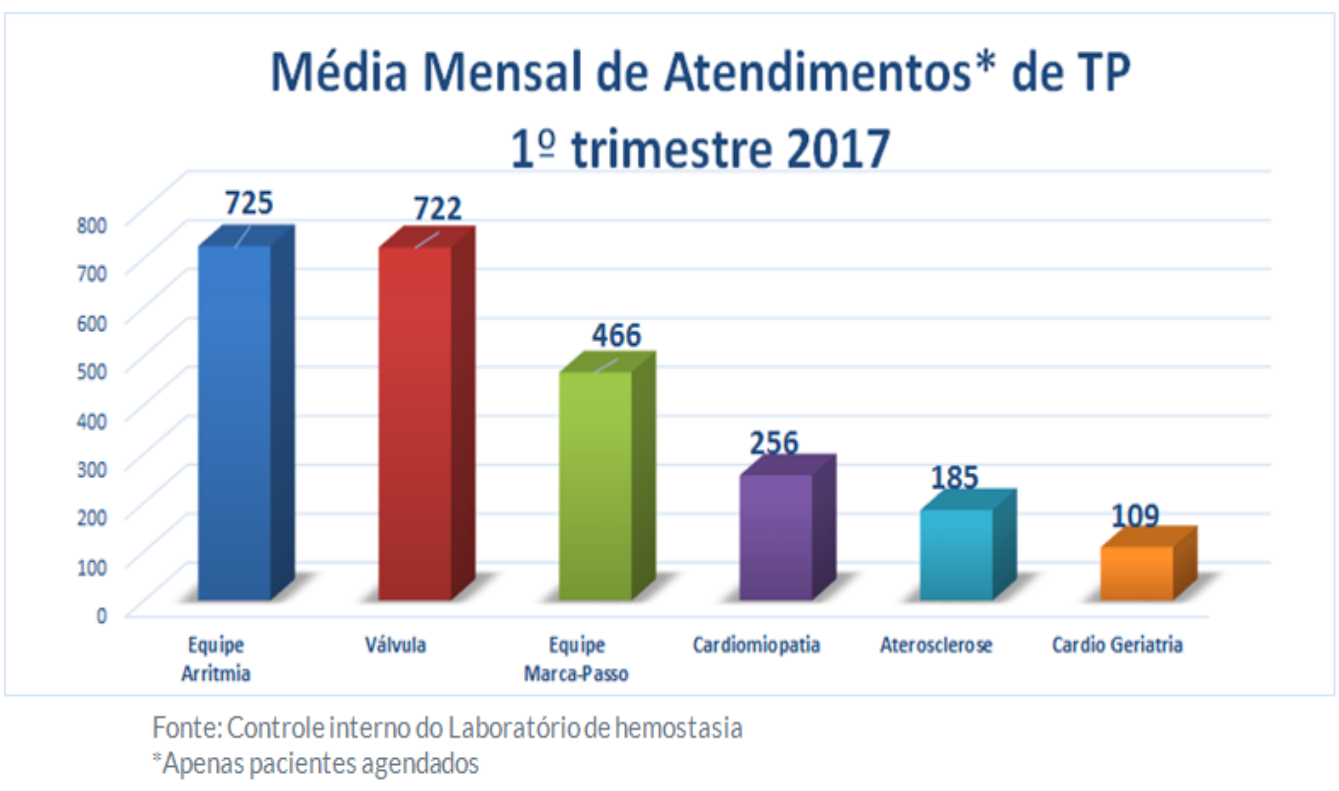

Gráfico 2. Média mensal de atendimentos de pacientes com coleta de TP agendada no $1^{\circ}$ trimestre de 2017.

As equipes de aterosclerose, arritmia, cardiomiopatia, marca-passo, cardiogeriatria e pesquisa acompanham, através do laboratório de hemostasia, os resultados dos exames dos pacientes, classificando-os em dentro ou fora da faixa terapêutica. No entanto, tal informação serve apenas para seguimento das equipes, não é classificada e contabilizada automaticamente no sistema.

Observando o controle de anticoagulação dos pacientes com coleta de TP agendados em 2016, notamos que $38 \%$ à $56 \%$ dos pacientes de cada grupo não estão dentro da faixa terapêutica. Os valores de resultados de exames de TP fora da faixa terapêutica estão descritos em detalhes no Gráfico 3. A equipe de arritmia apresentou a menor porcentagem de exames fora da faixa terapêutica, com $38 \%$, e a equipe de cardiomiopatia apresentou o maior percentual, com $56 \%$. 


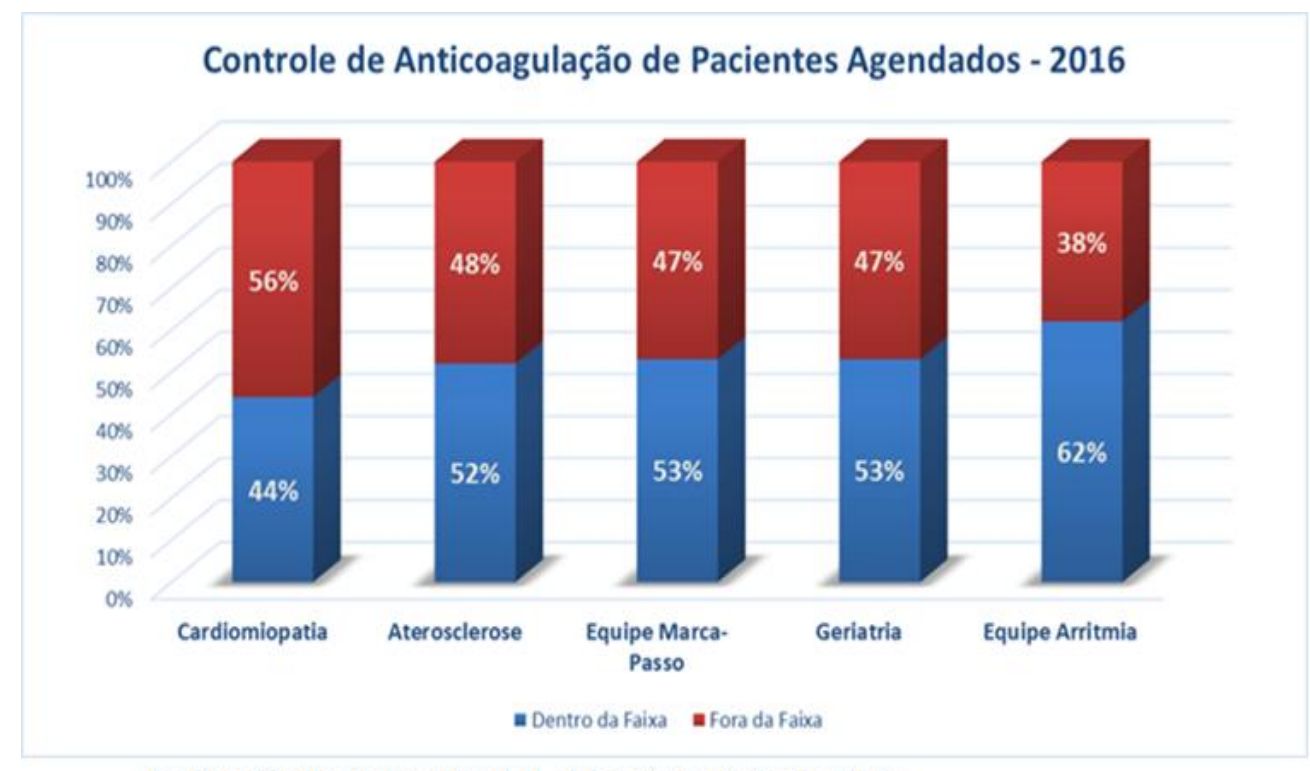

Fonte: Controle Interno do Laboratório de hemostasia

*Apenas pacientes agendados

Gráfico 3. Controle de anticoagulação de pacientes com coleta de TP agendada, em 2016.

Os pacientes que apresentem desvios nos resultados do TP que foi coletado durante $o$ atendimento ambulatorial agendado (e que não apresentem condições que justifiquem sua internação) são convocados para repetir o exame no intervalo de sete dias, para evitar riscos à saúde do paciente decorrentes do uso do medicamento. Considerando tal grupo de pacientes e incluindo o restante dos pacientes que passam pelo atendimento e realização do exame de TP, verificamos que entre $58 \%$ a $65 \%$ dos pacientes não estão dentro da faixa terapêutica, quando considerados os dados do primeiro trimestre de 2017. Os valores de resultados de exames de TP fora da faixa terapêutica estão descritos em detalhes no gráfico 4 . A equipe de aterosclerose apresentou o menor percentual de resultados fora do intervalo terapêutico, com $58 \%$, e a equipe de marca-passo apresentou o maior percentual, com $65 \%$ dos resultados fora do intervalo terapêutico. 


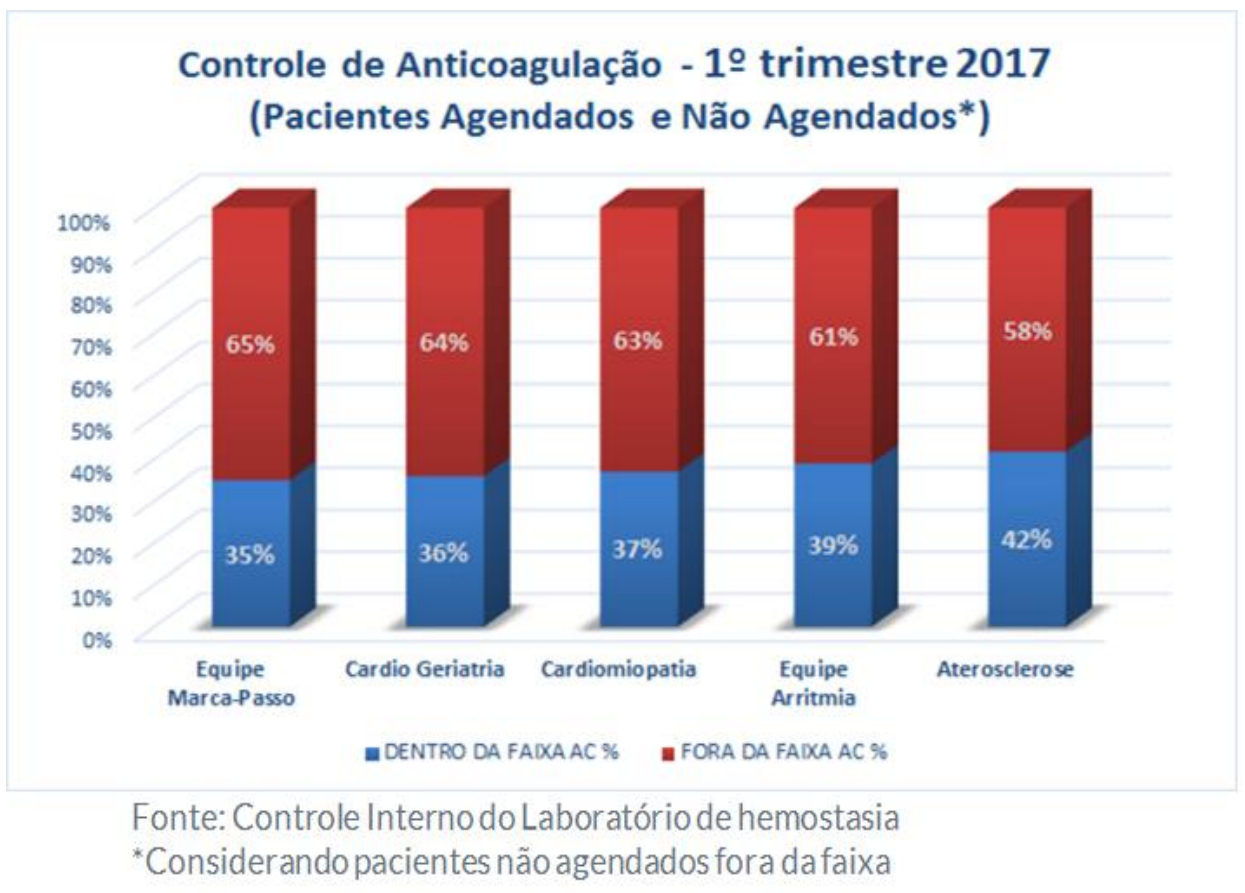

Gráfico 4. Controle de anticoagulação de pacientes com coleta de TP agendada e pacientes convocados para repetir exame, $1^{\circ}$ trimestre de 2017.

Não foi possível determinar quantas vezes cada paciente realizou o exame de TP de forma agendada no período analisado, uma vez que são registrados apenas os resultados dos exames no sistema do laboratório, porém não há controle e registro dos atendimentos agendados no sistema de prontuário da instituição, pois o contato com o médico e a conduta frente ao resultado do exame ocorre de forma informal nos intervalos das consultas ambulatoriais. No momento, as ausências de registro de dados inviabilizam análises mais elaboradas.

\section{Proposta de Intervenção}

A partir dos dados observados, diagnóstico situacional, levantamento bibliográfico e benchmarking realizado em um hospital especializado em cardiologia de São Paulo foi recomendado a implantação um serviço centralizado com a criação de um "ambulatório de anticoagulação", contendo as seguintes melhorias:

a) Sistematização e registro do exame de TP: medida de média a longo prazo, com inclusão no sistema de prontuário eletrônico próprio da instituição para registro e acompanhamento de anticoagulação, sendo uma aba personalizada, com fácil acesso e sem sistema de triagem, diferentemente da aba de consultas e prontuário eletrônico. Dentro desta sistematização, incluir o "alvo terapêutico" para diferenciar as especificidades do INR de cada grupo de cardiopatas e a sinalização de 
episódios de hemorragias e/ou riscos. Além disso, com a sistematização será possível levantar o histórico de resultado de exames de TP, histórico da dosagem medicação anticoagulante, extração de dados e indicadores de número de pacientes (incluindo a ficha SUS para contabilizar cada atendimento).

b) Tabela de anticoagulação do paciente (Figura 2): material informativo para o paciente, incluindo o alvo terapêutico, histórico dos exames de TP, INR, doses específicas de cada dia da semana, data de retorno e observações. Possivelmente com interface a aba de anticoagulação inserida no sistema citado acima.

c) Implantação do Point of Care: apenas para pacientes com restrições ou limitações, tais como: pessoas com dificuldades de locomoção ou alto grau de dependência (incluindo idosos), gestantes e crianças com dificuldade de coleta endovenosa. Para esta ação, considera-se agilização no fluxo do exame de TP pelo laboratório e melhor experiência do paciente, respeitando suas especificidades.

d) Atuação da equipe multiprofissional: medida de médio a longo prazo, com atendimento individual para os novos pacientes e pacientes fora da faixa terapêutica (dificuldade de controle de anticoagulação), incluindo a educação do paciente, com melhor orientação e adesão ao tratamento. $O$ atendimento inclui a equipe de enfermagem (realização da anamnese e orientação dos eventuais riscos e episódios hemorrágicos, farmácia (realização da interação medicamentosa e verificação da polifarmácia), nutrição (realização da orientação dietética e nutricionais), e o médico (realização do ajuste da dosagem da medicação e verificar conclusão da equipe multiprofissional). Para este atendimento, inclui-se o fluxograma abaixo.

e) Serviço de call center: medida de média a longo prazo, sendo um atendimento receptivo via telefone exclusivo para pacientes em anticoagulação para eventuais casos de emergência (principalmente para casos de hemorragias, incluindo uma comunicação direta com o serviço de ambulância caso seja necessário), introdução de nova medicação e realização de procedimentos. Este canal de atendimento é eficaz para evitar agravos e óbitos, sendo um modelo utilizado em outros países e verificado pelo Benchmarking realizado.

f) Introdução de novas medicações: inclusão de tratamento com novos medicamentos apenas para alguns pacientes de risco e sem adaptação ao medicamento varfarina. Apesar do custo elevado, há um maior controle da anticoagulação, resultando na diminuição da frequência de exames de TP e menor risco de entrada de urgência e emergência. 


\section{Alvo Terapêutico:}

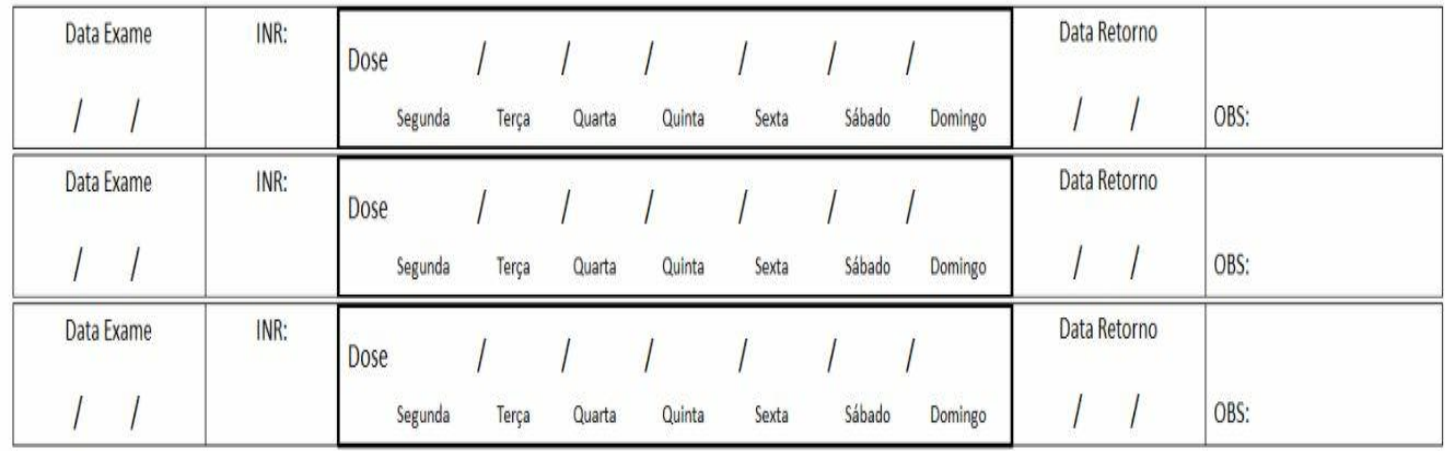

Figura 2. Modelo proposto da Tabela de Anticoagulação do Paciente.

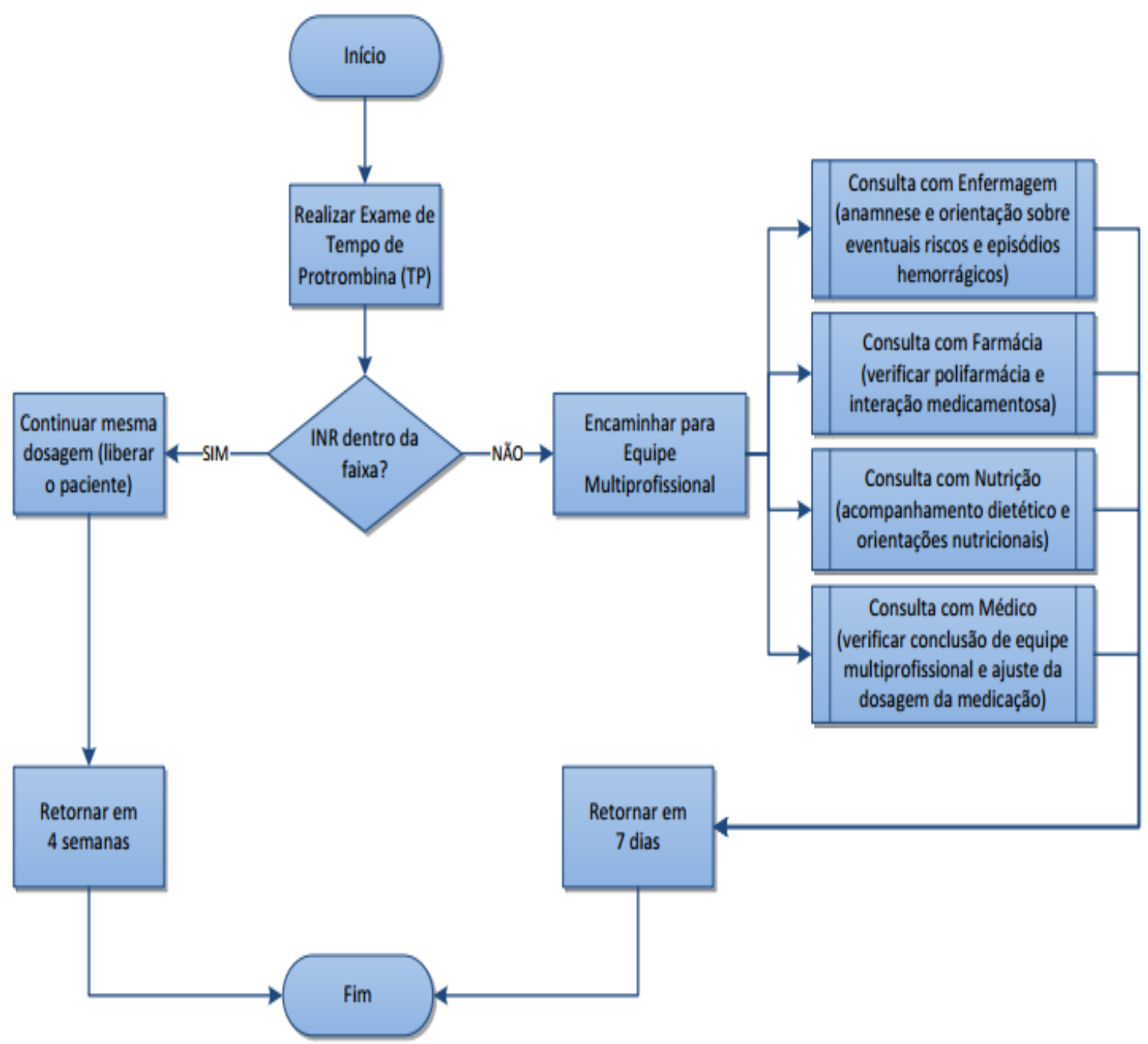


Figura 3. Fluxograma proposto para o atendimento multiprofissional dos pacientes em anticoagulação.

\section{DISCUSSÃO}

De acordo com os resultados observados, um benchmarking realizado em um hospital especializado em cardiologia de São Paulo e do levantamento bibliográfico, foi recomendado a implantação um serviço centralizado com a criação de um "ambulatório de anticoagulação". Este tipo de serviço já existe em alguns estados do Brasil 3,4,5,6,7, tais como: São Paulo, Rio de Janeiro, Minas Gerais, Bahia e Rio Grande do Sul e algumas experiências internacionais como: Estados Unidos ${ }^{8}$, Portugal ${ }^{1,9}$ e Colômbia ${ }^{10}$.

Conforme a experiência da Colômbia ${ }^{10}$, os benefícios de monitoramento pacientes em clínicas de anticoagulação incluem a diminuição das taxas fora da faixa terapêutica, redução de complicações hemorrágicas (diminuição dos atendimentos de emergência e menos hospitalizações por ano) e aumento do tempo de permanência dentro do intervalo terapêutico (cerca de $60-68 \%$ dos pacientes permanecem no tempo de intervalo terapêutico, enquanto aqueles que realizam o monitoramento ficam entre $51 \%$ e $57 \%$ do tempo).

Para Cruz e Campos ${ }^{9}$, de acordo com as experiências de Portugal, o ambulatório de anticoagulação parte dos seguintes pressupostos para funcionamento:

- Capacidade logística de atender com uma acessibilidade adequada a pessoas com mobilidade diminuída (idosos e cadeirantes), com instalações de consulta e sala de espera apropriadas.

- Capacidade técnica de execução das análises necessárias para a monitorização da anticoagulação oral, que implica a colheita de amostras apropriadas para a realização do TP e INR

- Capacidade de ter recursos humanos treinado e experiente na área da monitorização de anticoagulantes orais, incluindo equipe multiprofissional

Além disso, inclui-se a otimização da monitorização de anticoagulantes tais como: monitorização de anticoagulantes orais sistemática e coordenada entre a equipe multiprofissional; educação do doente de forma contínua, através de consulta presencial, de telefone ou internet/email, que seja acompanhada, de preferência, com informação escrita (folheto informativo); base de dados informática própria de registo das consultas médicas, que tenha capacidade de gerar informação escrita para os doentes (dosificação informática); capacidade de lidar com efeitos adversos da anticoagulação oral, com episódios hemorrágicos e trombóticos (acesso a vitamina $\mathrm{K}$, administrada via oral ou via endovenosa e ligação fácil a serviços de urgências); criação de protocolos de 
anticoagulação estabelecidos; capacidade de avaliação da qualidade da clínica de anticoagulação (controle anticoagulante e avaliação da satisfação dos pacientes).

\section{CONCLUSÃO}

Considerando que o TP é importante no contexto ambulatorial da cardiologia, pois além de monitorar o grau de anticoagulação do sangue de pacientes, é necessário seu acompanhamento para a utilização da medicação anticoagulante e de acordo com a situação atual que foi estudado, há uma necessidade do local e grande demanda do ambulatório para o serviço de anticoagulação, na qual necessitam mudanças e melhorias no processo de atendimento do paciente em anticoagulação, principalmente por ser um serviço especializado de alta complexidade e ter impacto em vários setores do ambulatório, na qualidade da assistência prestada pelos profissionais, na experiência do paciente e no controle da enfermidade.

Conversas realizadas com as partes envolvidas do instituto em relação ao trabalho desenvolvido, todos se mostraram receptivos as mudanças e há uma conscientização que o serviço e o processo do atendimento de TP precisa ser otimizado, para que seja viável entregar resultado em saúde.

\section{REFERÊNCIAS}

1. Barreira R, Ribeiro J, Farinha M, Martins R, Rodrigues I, Mendes Z, et al. Monitoring therapy with oral anticoagulants. Anticoagulation clinics vs assistant physician. Acta Médica Port. 2004;17(6):413-6.

2. DIRETRIZES BRASILEIRAS DE ANTIAGREGANTES PLAQUETÁRIOS E ANTICOAGULANTES EM CARDIOLOGIA [Internet]. Programas de Pósgraduação em Ciências da Saúde: Cardiologia. [citado 13 de dezembro de 2017]. Disponível em: http://www.ppgcardiologia.com.br/

3. Costa JM, Pimenta M de C, Antunes MIS da S, Costa MA, Martins MAP. IMPLANTAÇÃO DE UM AMBULATÓRIO DE ANTICOAGULAÇÃO EM UM HOSPITAL DE ENSINO: ESTUDO DESCRITIVO. Rev APS [Internet]. 3 de dezembro de 2015 [citado 13 de dezembro de 2017];18(1). Disponível em: https://aps.ufjf.emnuvens.com.br/aps/article/view/2400

4. Toledo ISB de, Costa JM, Fontes CB, Souza MM de, Groia RC de S, Machado CJ, et al. ENTENDIMENTO DE PACIENTES SOBRE PARTICIPAÇÃO EM OFICINAS EDUCACIONAIS EM UM AMBULATÓRIO DE ANTICOAGULAÇÃO. Rev APS [Internet]. 20 de janeiro de 2017 [citado 13 de Dezembro de 2017];19(3). Disponível em: https://aps.ufjf.emnuvens.com.br/aps/article/view/2574 
5. Bosa MCP. Anticoagulação ambulatorial monitorada por consulta de enfermagem: fatores influentes às alterações clínicas e laboratoriais em portadores de fibrilação atrial em uso de varfarina: estudo de caso-controle aninhado. 2012 [citado 13 de dezembro de 2017]; Disponível em: http://localhost:8080/jspui/handle/1/1145

6. 698---Estrategias-para-promocao-da-adesao-em-um--ambulatorio-deanticoagulacao-contribuicao-para-a-efetividade-do--tratamento---Formatado--1159-1177.pdf [Internet]. [citado 13 de dezembro de 2017]. Disponível em: http://www.rbfarma.org.br/files/698---Estrategias-para-promocao-da-adesaoem-um--ambulatorio-de-anticoagulacao-contribuicao-para-a-efetividade-do-tratamento---Formatado---1159-1177.pdf

7. Silva SB da, Gonçalves NS, Santos DC. Implantação de um modelo de descentralização de auditoria de contas hospitalares em um hospital de grande porte na região sul do Brasil. Rev Adm em Saúde [Internet]. 17 de outubro de 2017 [citado 13 de dezembro de 2017];17(69). Disponível em: http://www.cqh.org.br/ojs-2.4.8/index.php/ras/article/view/58

8. Errichetti AM, Holden A, Ansell J. Management of Oral Anticoagulant Therapy: Experience with an Anticoagulation Clinic. Arch Intern Med. 1 de Outubro de 1984;144(10):1966-8.

9. Clínicas de anticoagulação, situação actual e perspectivas futuras ScienceDirect [Internet]. [citado 13 de dezembro de 2017]. Disponível em: http://www.sciencedirect.com/science/article/pii/S0870255112700409

10. Taboada LB, Silva LE, Montenegro AC. Beneficios de la clínica de anticoagulación. Acta Méd Colomb. Outubro de 2013;38(4):239-43.

Recebido: 21 de fevereiro de 2019. Aceito: 18 de março de 2019.

Correspondência:Ticiane Nishimoto. E-mail: tici.nishimoto@gmail.com

Conflito de Interesses: os autores declararam não haver conflito de interesses. 
(C) This is an Open Access article distributed under the terms of the Creative Commons Attribution License, which permits unrestricted use, distribution, and reproduction in any medium, provided the original work is properly cited 